\title{
Einige Beobachtungen zu gemeinsamen Motiven in Hartmanns Erec und Wolframs Parzival
}

\section{KATHRYN SMITS}

Dieser kurze Aufsatz hat ein eng umrissenes Thema und ein bescheidenes Ziel. Vor einigen Jahren hat Rüdiger Schnell überzeugend nachgewiesen, daß es in Wolframs Parzival eine Reihe von Anklängen und Anspielungen gibt, die darauf hindeuten, daß Wolfram sich sehr genau mit dem Hartmannschen Erec -Roman auseinandergesetzt und sich von $\mathrm{ihm}$ hat anregen lassen. ${ }^{1}$ Ich möchte hier an Schnells Ergebnisse anknüpfen und auf einige weitere Parallelen und Kontraste aufmerksam machen, die mir bei der Beschäftigung mit Erec und Parzival aufgefallen sind. Im Grunde untermauern sie, was schon er festgestellt hat, nämlich daß es sich nicht um eine systematische Nachfolge handelt, sondern um die kontrastierende oder parallelisierende Verarbeitung einzelner Motive, die bei Wolfram zum Teil für ganz andere Gestalten oder in anderen Situationen verwendet werden. Wir erleben zur Zeit eine erstaunliche Hartmann-Renaissance; es vergeht kaum ein Monat, in dem nicht irgendwo im deutsch-oder englischsprachigen Raum zum Armen Heinrich, zu Erec, Iwein oder Gregorius Stellung genommen wird. ${ }^{2}$ So werden die Gestalten in diesen Romanen immer wieder neu beleuchtet, und es öffnen sich dadurch auch für die vergleichende Betrachtung neue Perspektiven. Ein Beispiel wäre die Erkenntnis, die sich in den letzten Jahren allgemein durchgesetzt hat, daß die Hartmannsche ErecGestalt (im Gegensatz zum Erec Chrestiens) zu Beginn des Romans ein in vieler Hinsicht unreifer junger Mann ist; zur Zeit des großen Hochzeitsturniers (Er. 2222ff.) hat seine Entwicklung, trotz des ihm vom Dichter gespendeten Lobes, noch kaum begonnen. ${ }^{3}$ Wenn wir nun aber Erec als eine reifende, sich erst allmählich entfaltende Gestalt betrachten, so drängt sich mehr als früher der Vergleich mit Parzival auf. Die sehr wesentlichen Unterschiede springen zuerst ins Auge, wir sollten uns aber auch fragen, ob der Entwicklungsprozeß des einen mit dem des anderen sinnvoll zu vergleichen wäre. Gerade die Beschäftigung mit Ähnlichkeiten oder auffallenden Kontrasten kann uns zu Motiven führen, die beide Dichter beschäftigten und die somit eine ïber das Einzelwerk hinausreichende Bedeutung haben könnten.

Der Werdegang des jungen Erec weist tatsächlich manche Ähnlichkeiten mit dem des jungen Parzival auf. Beide sind sie Königssöhne, durch Geburt und 
Veranlagung zum Rittertum bestimmt. Beide sind sie jung und werden nach vielen Abenteuern das ihnen im Rahmen ihrer Geschichte gesteckte hohe Ziel erreichen. In beiden Fällen ragt dieses Ziel über die höfischen, von der Artuswelt verkörperten Idealwerte hinaus, namentlich dadurch, daß es, sowohl im Erec als auch im Parzival, religiöse Werte umfaßt, die der Artuswelt fremd sind. Beide Hauptgestalten werden von Anfang an als vielversprechend dargestellt: Die menschliche Integrität der beiden steht nie in Frage, obwohl ihr Verhalten keineswegs immer richtig ist. Wie Parzival erwirbt auch der junge Erec als erstes den äußeren Glanz ritterlicher Erfolge und die ehrenvolle Zugehörigkeit zum Artuskreis. Die Körperkraft der beiden zeigt sich bald; das Tragen der Rüstung und die Handhabung der Waffen im Kampf werden ihnen bald zur Selbstverständlichkeit. Auf dieser Ebene der Kommunikation mit dem Mitmenschen sind sie ihrer Sache von Anfang an sicher. Wo es um Kampf, Aggression, Draufgängertum geht, erfüllen sie schon früh die Erwartungen ihrer Umwelt.

An Parzival, dem nicht höfisch Erzogenen, wird das besonders deutlich in Begegnungssituationen dargestellt. Im Umgang mit Menschen ist körperliche Aggression sehr lange, bis ins neunte Buch, sein wichtigster Verhaltensmaßstab. Jeschutes Ring, Kuß und Spange nimmt er sich mit roher Gewalt ( $\mathrm{Pa}$. 130, 26ff.), für Sigune möchte er sich kämpfend einsetzen (Pa. 141,25 - 142,1). Die Mißhandlung Cunnewares hätte er, wäre das möglich gewesen, sofort mit Gewalt gerächt (Pa. 153, 18ff.), das Gespräch mit Ither läßt sich für ihn nur mit dem gabilot sinnvoll führen (Pa. 153,21ff.). Auch Condwiramurs erlöst und gewinnt er durch Kampf (Pa. 180,20ff.). Wenn sich der unbewaffnete Parzival auf Munsalvaesche durch Worte beleidigt fühlt, reagiert er mit einer verständnislosen Aggression, die seine innere Unsicherheit verrät (Pa. 229,1ff.). Der Situation auf der Gralsburg, wo die Mitleidsfrage von ihm erwartet wird, ist Parzival noch nicht gewachsen, er ist aber gern bereit, seinem Gastgeber kämpfend zu dienen ( $\mathrm{Pa} .246,11 \mathrm{ff}$.). Auch wo er angetanes Leid wieder gut macht, tut er das auf dieser Stufe seiner Entwicklung nur dort, wo es unter Anwendung körperlicher Aggression möglich ist: Jeschutes ere wird durch den Kampf mit Orilus wiederhergestellt ( $\mathrm{Pa} .258,1 \mathrm{ff}$.), durch den Sieg über Keie wird Cunneware gerächt (Pa. 293,19ff.). ${ }^{4}$ Das Ablegen von Rüstung und Waffen in der Höhle des Trevrizent ist eine symbolische Handlung, mit der diese Lebensphase Parzivals zu Ende geht. Wenn er sie später wieder anlegt, hat sich sein Gottesverständnis, und damit auch sein Selbstverständnis, gewandelt. ${ }^{5}$

Der höfisch erzogene junge Erec verhält sich weitaus zivilisierter als Parzival. Im Umgang mit Menschen, sei es mit der Königin, sei es mit dem ihm grob begegnenden Ither, benimmt er sich nach allen Regeln der Gesellschaft. Es gibt in Hartmanns Erec keine vergleichbare Kette von aggressiven Handlungen; 6 Erec beherrscht und versteht ja die Sprache und Gestik der höfischen Welt, und bei Parzival führt gerade die Unkenntnis dieser Kommunikationsmittel sehr häufig zu Mißverständnissen und Fehlreaktionen. Bezeichnenderweise ist die 
Trevrizent-Begegnung ein einziges langes Gespräch, in dem Parzivals Ohren geöffnet und seine Zunge gelöst werden, während er auf Musalvaesche nur schweigend, verständnislos schauen konnte. Dem Erec ist diese Problematik unbekannt. Trotzdem sind auch für ihn Rüstung und Waffen in ähnlicher Weise symbolisch. Auch Erec ist innerlich unsicher; das ihm von Rüstung und Waffen verliehene Selbstbewußtsein ist trügerisch. Das wird einmal kurz, aber eindringlich gezeigt. Wenn er, habelos (Er. 238) und bloz als ein wip (Er.103), im Gemäuer bei Tulmein unerwartet Koralus vorfindet, ist Erec vollkommen verunsichert. Er verliert nicht die Fassung, wie es Parzival in Versen Pa. 229,1ff. tut, denn Erec weiß durch höfische Gesten und Worte eine Brücke zum anderen zu schlagen:

$$
\begin{aligned}
& \text { Er. } 298 \text { sin hende habete er vür sich, } \\
& \text { einem wol gezogenem manne gelich, } \\
& \text { und gienc da er den alten sach. }
\end{aligned}
$$

Innerlich ist er aber voller Unsicherheit und Scham:

Er. 301 mit zwivel er zuo im sprach:

'herre, mir waere herberge not.'

diu bete machete in schamerot.

Verschämtes Verhalten sollte an und für sich positiv bewertet werden (Pa. 170,16f. ir sult niemer iuch verschemen./verschamter lip, waz touc der mer? ), in dieser Szene handelt es sich aber nicht um die zartfühlige Scheu, die einen reifen höfischen Menschen im Umgang mit anderen auszeichnet, sondern Erecs schame gilt der eigenen Person. Hier wird ein junger Mann dargestellt, der zum erstenmal in seinem Leben ohne die äußeren Abzeichen des Ranges (Geld und Gut), der Manneswürde (Rüstung und Waffen) und dazu noch ganz allein einem fremden Menschen entgegentreten muß, dessen Reaktion er nicht sicher sein kann. Einen Augenblick sehen wir Erec hier bloz und erkennen seine innere Unsicherheit. Parzival wird, in Versen $\mathrm{Pa}$. 229,1-22, in einer vergleichbaren Lage dargestellt. Er hat bei Gurnemanz vieles gelernt, hat zu Pelrapeire seinen Wert als Kämpfer erwiesen und hat Condwiramurs geheiratet. Äußerlich steht es gut um ihn. Nun befindet er sich auf Munsalvaesche, man hat ihn von seinem Schwert getrennt. Die Vorladung zum inzwischen eingetroffenen Schloßherrn wird in einer Weise ausgesprochen, die Parzival in seiner Unerfahrenheit falsch interpretiert, er reagiert mit stummer Wut, die fast in körperliche Aggression umschlägt:

Pa. 229,10 do er sin swert wol gemal

ninder $b i$ im ligen vant,

zer viuste twanc er sus die hant,

daz daz bluot uz den nageln schoz

und im den ermel gar begoz.

15 'nein, herre!' sprach diu ritterschaft, 
'ez ist ein man der schimphes kraft

hat. swie trurec wir anders sin,

tuot iuwer zuht gein im schin:

ir ensultz niht andern han vernomen,

20 wan daz der vischaere si komen.

dar get (ir sit im werder gast)

und schütet ab iu zornes last.'

Auch hier wird, wie beim jungen Erec, die innere Unsicherheit des Helden in einem kritischen Moment kurz, aber grell beleuchtet. Die impulsive Reaktion wird, mit Hilfe der ritterschaft, welche Geste und Sprache des redespaehen mannes ( $\mathrm{Pa} .229,4)$ besänftigend interpretiert, sehr schnell überwunden. Sie bleibt uns aber in Erinnerung. ${ }^{7}$ Auch für Erec normalisiert sich die Lage fast sofort. Koralus ist, wie sich herausstellt, zwar arm, aber höfisch und sogar ein Freund des Vaters. Durch ihn wird Erec jetzt der Einstieg in die ritterliche Welt des Kampfes ermöglicht: Wie verschieden Erec und Parzival auch reagieren mögen, wie anders die beiden Erzählungen sich auch entwickeln, diese beiden Szenen haben dieselbe Funktion. Sie zeigen, daß es dem jeweiligen jungen Mann an innerer Reife und Sicherheit fehlt, und sie deuten auf sein Verhalten in der bevorstehenden Krise hin. Wichtig ist weiter, daß sowohl Erec als auch Parzival bald darauf mit einer Situation fertig werden müssen, die nicht mit dem Schwert bewältigt werden kann, und in der der Held scheitert. In beiden Fällen handelt es sich hier um eine mitmenschliche Situation: Parzival reagiert falsch auf das Leiden Anfortas', Erec reagiert falsch auf die Ehe.8

Rüdiger Schnell hat auf gewisse Parallelen zwischen Hartmanns Erec und Wolframs Gawan hingewiesen. ${ }^{9}$ Interessant ist daneben auch der Vergleich zwischen Wolframs Gawan und Hartmanns Guivreiz-Gestalt. Wir beschränken uns dabei wieder auf einige wenige Motive. Gawan ist mit Parzival verwandt, er ist ihm ebenbürtig, er ist ein ebenso guter Kämpfer wie er, er ist sein Freund. Die höfische Form, mit der sich Parzival so lange abmüht, beherrscht er vollkommen. Gawans Abenteuer finden auf einer anderen Ebene statt als die Parzivals: Er kennt weder dessen quälende zwivel noch widerfährt ihm etwas, was Parzivals Erhöhung zum Gralskönig vergleichbar wäre. Guivreiz ist mit Erec nicht verwandt, er ist ihm aber ebenbürtig, und Erec nimmt ihn nicht als Vasallen, sondern als Freund an (Er. 4439ff.). Auch Guivreiz ist ein vollkommen höfischer Mann.10 Er und Erec kämpfen zweimal, wobei jeder einmal siegt (der geschwächte Erec befindet sich beim zweiten, von ihm verlorenen Kampf eindeutig im Nachteil). Schon oberflächlich erinnert der im Morgengrauen stattfindende 'Bruderkampf' zwischen Parzival und dem verwundeten Gawan (Pa. 679,1ff) an den nächtlichen Zusammenstoß zwischen Guivreiz und dem schwer verletzten Erec (Er. 6862ff.). In beiden Fällen wird, wie so oft in den 
Artusromanen, im letzten Augenblick eine Katastrophe verhindert. Es gibt aber auch viele Unterschiede. Guivreiz ist absichtlich ausgeritten, um Erec beizustehen, und meint einem Feinde Erecs zu begegnen; Gawan meint, auf Gramoflanz zu stoßen, und es fällt ihm überhaupt nicht ein, daß dieser fremde Ritter etwas mit Parzival zu tun haben könnte. Bei Hartmann ist Erec der Verletzte und schließlich der Besiegte; bei Wolfram ist es Parzival, der den durch Wunden geschwächten Freund besiegt. Auch die Funktion dieser Szenen innerhalb der Erzählung ist jeweils eine andere. Viel wichtiger als das alles ist die innere Haltung des Guivreiz bei der ersten Begegnung mit Erec (4320ff.). Es lassen sich hier interessante thematische Beziehungen zu Gawans Verhalten in der Blutstropfenszene (Pa. 299,27ff.) erkennen. Äußerlich sieht alles ganz anders aus. Der Kontrast zwischen diesen beiden Szenen ist, bei näherer Betrachtung, geradezu auffallend. Erec reitet in Begleitung seiner Gattin, steht ihr aber innerlich fern; Parzival reitet allein, ist aber im Herzen so sehr mit der fernen Gattin beschäftigt, daß er seine Umgebung nicht mehr wahrnimmt. Das Auftreten Guivreizs führt zu einem schweren Kampf; Gawans Erscheinen dagegen verhindert einen solchen Kampf. Zwei Grundmotive haben diese Szenen aber gemein: (a) In ihrem Mittelpunkt steht das Verhältnis des Helden zu seiner Ehefrau, und (b) es ist der Freund (bzw. der sich rasch zum Freund entwickelnde Gegner), der diese Lage aufgrund seiner eigenen positiven Gesinnung sofort durchschaut und sie zum Ausgangspunkt seines Handelns macht.

Alle Männer, denen Erec und Enite auf ihrer aventiure-Fahrt bisher begegneten, haben auf Enite 'falsch' reagiert. Die schoene Enitens, die ihr 'Frau-Sein' nach außen hin manifestiert und die an und für sich weder etwas Positives noch etwas Negatives ist, ${ }^{11}$ hat in den Räubern nur Habsucht und im ersten Grafen eine falsche minne ausgelöst, die sich ebenfalls in Habsucht äußerte und ihn für die wahre Beziehung zwischen Erec und Enite blind machte. Die positive Kehrseite dieser von Enite unbewußt ausgelösten zerstörerischen minne ist ihre tiurende kraft; diese gilt nur dem geliebten Ehemann. Erec ist sich dessen zu diesem Zeitpunkt noch nicht bewußt und erlaubt Enite nicht, die ihr zustehende Rolle als Ehefrau zu spielen. ${ }^{12}$ Enite selbst.weiß nicht um die sowohl positive als auch verderbliche Wirkung ihrer Person auf Männer. Sie reagiert gefühlsmäßig: Auf Erec reagiert sie mit bedingungsloser triuwe, auf die anderen Männer mit Abwehr. ${ }^{13}$ Guivreiz begegnet dem Ehepaar zu einem Zeitpunkt, da Enitens triuwe sich dem Hörer/Leser schon stark eingeprägt hat und da Erecs Zorn seinen Höhepunkt erreicht hat (Er. 4263f. sin zorn wart groz und ungemach/und unsenfter dan e.). Jeder Mann hat bisher auf Enitens schoene als isoliertes Phänomen reagiert, Guivreiz aber nimmt den inneren Zusammenhang wahr. Es spielt für ihn offensichtlich keine Rolle, daß die Harmonie zwischen Erec und Enite gestört ist (das, und nur das, war dem ersten Grafen aufgefallen!), sondern er sieht das Entscheidende: Die schoene Enitens gilt ihm als Garantie für Erecs Wert als Mann und Ritter: 


$\begin{aligned} & \text { Er. } 4329 \text { mich bedunket ane strit, } \\ & \text { ir muget wol ein degen sin. } \\ & \text { daz ist an zwein dingen schin: } \text { ir vüeret, sam mir min lip, } \\ & \text { daz aller schoeniste wip } \\ & \text { der ich ie künde gewan: } \\ & 4335 \text { wer gaebe die einem boesen man? } \\ & \text { dar zuo sit ir gewafent wol, } \\ & \text { als ein guot ritter sol. . . 14 }\end{aligned}$

Guivreiz fordert Erec nicht auf, seine Frau zu verteidigen, sondern sich im Kampf um das eigene Leben und die Ritterehre von der Frau inspirieren zu lassen: Er. 4376f. wert iuch durch iuwer schoenez wip, /welt ir behalten den lip. Der tiurende Einfluß der Frau soll nicht nur die Kampftüchtigkeit des Gegners, sondern auch dessen Barmherzigkeit steigern:

$\begin{aligned} \text { Er. } 4439 & \text { Erec fil de roi Lac } \\ & \text { hate nach missetan, } \\ & \text { wan er wolde in erslagen han. } \\ & \text { 'nein', sprach er, 'ritter guot, } \\ \text { durch dinen tugenthaften muot } & \text { unde durch din schoene wip } \\ 4445 & \text { so la mir den lip } \\ & \text { und ere got an mir. }\end{aligned}$

Nicht die oberflächlichen Erscheinungen - die Schönheit der Frau; die gestörte Harmonie im Verhältnis der beiden, die sich eventuell ausnutzen ließe - sind für Guivreiz wichtig. Er sieht nicht nur; er versteht, was er sieht, und versteht somit mehr als Erec selbst. Gerade darin berührt er sich mit Gawan. Auch Gawan sieht: Seine Augen sehen die erstarrte Haltung Parzivals (diese, und nur diese, war Keie aufgefallen!). So wie Guivreiz den verborgenen Einfluß Enitens auf Erec wahrnimmt, so versteht auch Gawan den tieferen Sinn hinter dem, was seine Augen sehen:

Pa. 301,21 do dahte min her Gawan:

'waz ob diu minne disen man

twinget als si mich do twanc,

und sin getriulich gedanc

der minne muoz ir siges jehen?'

Gawan bedeckt die Blutstropfen und führt damit Parzival zur Normalität des Alltags zurück; Guivreiz fordert den Gegner zu einem ehrenvollen Kampf auf, in dem dieser zum erstenmal die Frau bewußt als die Quelle seiner männlichen Tugenden verstehen soll. Das sind sehr unterschiedliche Handlungen, sie sind aber, den Umständen entsprechend, in gleicher Weise positiv zu bewerten. Sowohl Guivreiz als auch Gawan legen hier eine tiefere Einsicht in die minne- 
Problematik an den Tag als der Held selbst; sowohl Guivreiz als auch Gawan helfen dem anderen aufgrund dieser Einsicht einen Schritt weiter, statt ihn selbstsüchtig auszunutzen. Auch die negativen Gegengestalten zu Guivreiz (der erste Graf) und zu Gawan (Keie) weisen in diesen Episoden entsprechende Parallelen auf. Diese beiden haben nur Augen für das Äußere, Oberflächliche. Der erste Graf nimmt nur wahr, daß zwischen Erec und Enite etwas nicht stimmt; diese Beobachtung führt zum Wunsch, die Frau selbst zu besitzen:

Er. 3770 wer gap iuch armen solhem man

der enmac noch enkan

iuch geren ze rehte?

Und später zu Erec:

Er. 4188
ez möhte an dirre vrouwen
ein tore wol schouwen
daz si iu niht enist ze maze.
welt ir daz ich iu laze,
arger schalc, den lip,
so lat beliben daz wip.

Keie sieht den erstarrten, von den'Blutstropfen gebannten Parzival, schließt daraus sofort auf sträfliche Trägheit und reagiert dementsprechend:

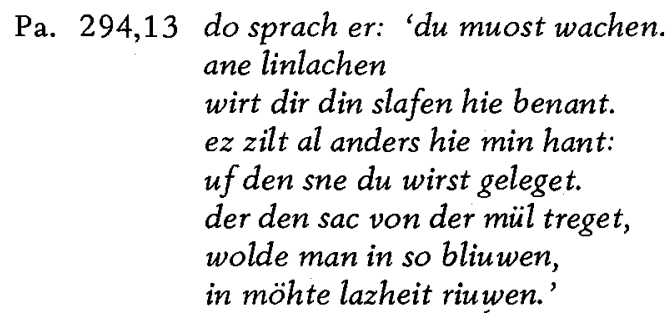

Sowohl der erste Graf als auch Keie tragen im folgenden Kampf nicht-tödliche Verletzungen davon (beide brechen den Arm: Er. 4214, Pa. 295,24). Auffallend ist weiter, daß Hartmann und Wolfram an derselben strategischen Stelle einen Minneexkurs einflechten, in dem von der gefährlichen Macht der Minne die Rede ist. Im Erec steht er in Versen 3694-3721, als der erste Graf sich zum Eingreifen anschickt, im Parzival in Versen 291, 1-293, 18, als Keie sich auf den Weg macht, um Parzival Mores zu lehren. Wie grundverschieden auch im einzelnen die Problematik dieser Szenen sein mag, sie haben diese minne-Thematik gemein, und es sieht ganz so aus, als hätte sich Wolfram bewußt, zum Teil kontrastierend, zum Teil parallelisierend, auf Erec bezogen.

Rüdiger Schnell hat interessante Kontraste zwischen dem Liebesverhältnis Erecs und Enitens und dem Parzivals und Condwiramurs aufgedeckt. ${ }^{15}$ Manches in seinem Aufsatz, was sich auf die Erec-Ehe bezieht, interpretiere ich 
anders, 16 ich teile aber seine Ansicht, daß es sich bei den minne-Erlebnissen Parzivals durchaus um "eine beabsichtigte Kontrastierung zu Hartmanns Erec" handeln könnte, und daß die Enwicklungsstufe, die Erec und Enite erst am Ende ihrer Geschichte erreichen, für Parzival und Condwiramurs in mancher Hinsicht den Ausgangspunkt bildet. ${ }^{17}$ Das Verhältnis zwischen Erec und Enite ist zu Anfang sehr stark sinnlich-physisch bedingt; die beiden sind so sehr auf die körperliche Nähe des Partners angewiesen, daß Erec es nicht mehr fertig bringt, in die Welt hinauszuziehen, um seinen ritterlichen Verpflichtungen nachzukommen. Die beiden lernen erst im Laufe der Handlung, sich von einander frei zu machen. In Parzivals Verhältnis zu Condwiramurs ist diese räumliche Bewegungsfreiheit des Mannes von Anfang an eine Selbstverständlichkeit: Sie beruht auf einer tiefen Einsicht Condwiramurs in das Wesen der ehelichen triuwe, wie sie im Erec-Enite-Verhältnis erst recht spät zustande kommt.18 Diese Beobachtungen Schnells können anhand eines weiteren gemeinsamen Motives der beiden Romane, mit dem er sich nicht befaßt, bekräftigt werden. An vielen Stellen im Parzival wird dargestellt, wie die tiurende Einwirkung der geliebten Ehefrau räumliche Entfernungen zu überwinden vermag, wenn der Mann es versteht, diese Ausstrahlung der Frau richtig zu 'empfangen'. Im Erec wirkt sich der tiurende Einfluß der Frau mehr oder weniger unmittelbar auf die Kampftüchtigkeit des Mannes aus, im Parzival wird dieses Motiv wesentlich subtiler behandelt. Trotzdem ist es auch dort von großer Wichtigkeit; man $k$ önnte sogar sagen, daß die Bedeutung Condwiramurs für den einsam umherirrenden Parzival erst voll verständlich wird, wenn man auch die 'Vorstufe', die Ehe von Hartmanns Erec und Enite, kennt.19 Erec bringt es zunächst überhaupt nicht fertig, sich von der Frau zu lösen (verligen-Szene, Er. $2924 \mathrm{ff}$ ); bei der folgenden aventiure-Fahrt bleibt es dann für ihn - obwohl er die Ehegemeinschaft, so weit ihm das möglich ist, verneint - absolut notwendig, daß Enite bei seinen Kämpfen körperlich zugegen ist. Schon beim Iders-Kampf hatte ihr Anblick seine Kräfte verdoppelt:

$\begin{aligned} \text { Er. } 935 & \text { und als er dar zuo ane sach } \\ & \text { die schoenen vrouwen Eniten, } \\ & \text { daz half im vaste striten: } \\ & \text { wan da von gewan er do } \\ & \text { siner krefte rehte } z \text { wo. }\end{aligned}$

Beim Turnier bewährt er sich glänzend, obwohl Enite dort nicht zugegen ist. Entscheidend ist aber, daß es sich dort um Ritterspiele, nicht um den tödlichen Ernstfall handelt. ${ }^{20}$ In dieser Turnierepisode werden Erecs Mut und sein Können im Waffenhandwerk zur Schau getragen; auch sie spielen bei den ihm bevorstehenden Kämpfen eine äußerst wichtige Rolle. Hier handelt es sich um die Kunst der körperlichen Aggression, die Erec, wie wir schon sahen, von Anfang an beherrscht. Dort aber, wo Erec sich allen Ernstes mit Gegnern messen muß, kommt etwas Entscheidendes dazu, dort gibt letzten Endes immer die 
tiurende kraft von Enitens minne den Ausschlag. Enite ist bei den Räuberkämpfen und beim Kampf mit dem ersten Grafen zugegen. Bei der ersten Begegnung mit Guivreiz wird Erec - der sich dieser Einwirkung der Frau auf ihn bisher nicht wirklich bewußt war - ausdrücklich vom Gegner darüber belehrt, welche Rolle die Frau beim Kampf für ihn spielt. Erec lernt hier etwas dazu. Vielleicht ist das der Grund, weshalb er den Kampf mit den Riesen, wenn auch mit knapper Not, ohne Enite besteht. Allerdings stürzt er ihr danach vür tot (Er. 5738) zu Füßen. Den Kampf gegen Oringles und seine Leute führt er wieder in Enitens Gegenwart. Der versehentliche 'Bruderkampf', in dem Erec von Guivreiz geschlagen wird, gehört nicht in die Reihe der Bewährungskämpfe, auch wenn Enite hier eine entscheidende Rolle spielt. Sehr wichtig ist dann wieder der Zweikampf zu Brandigan, dem Enite ausdrücklich ferngehalten wird, während die Dame Mabonagrins in Person anwesend ist. Die Ritter, die es gegen Mabonagrin aufgenommen haben, mußten dieses Handicap immer in Kauf nehmen; bisher ist jeder von ihnen daran gescheitert. 21 So wird in diesem Kampf gerade auch das richtige Verhältnis zwischen Erec und Enite auf die Probe gestellt. Enitens minne (Er. 8870, 9184), die die körperliche Trennung überwinden kann, ist in diesem Kampf nicht weniger wichtig als Erecs Mut und Beherrschung des Waffenhandwerks. Der Mabonagrin-Kampf 'befreit' demnach auch Erec endgültig von der körperlichen Bindung an die Frau: Von jetzt an braucht er sie, um die tiurende kraft ihrer minne auf sich einwirken zu lassen, nicht mehr in seiner unmittelbaren Nähe zu haben. Die Problematik, die zum verligen führte, ist damit überwunden. Erec und Enite haben damit die Stufe erreicht, auf der Parzival und Condwiramurs schon standen, da sie sich, als Auftakt zu Parzivals Gralserlebnis, von einander trennten. 22

Die Beobachtungen, die wir hier machen konnten, bestätigen einerseits schon Bekanntes: Wolfram hat sich intensiv mit Hartmanns Erec beschäftigt und sich zum Teil sehr kritisch mit ihm auseinandergesetzt, er hat Motive dieses Romans im Parzival aufgegriffen und weiterentwickelt. Andererseits erlauben uns diese Beobachtungen auch neue Ansätze, vor allem in der Erec -Interpretation. Motive, die offensichtlich von Wolfram als so interessant und wichtig empfunden wurden, daß er sie entweder parallelisierend oder kontrastierend selbst verarbeitete, verdienen zumindest unsere volle Aufmerksamkeit. Das gilt vor allem dort, wo Hartmann stark von Chrestien abweicht. Ein solches Motiv ist die tiurende kraft der minne der Ehefrau, die sowohl im Erec als auch im Parzival engstens mit dem Thema der weiblichen Schönheit und mit der allmählichen Entwicklung des Helden verbunden ist. Zu diesem Themenkomplex läßt sich noch manches sagen. Das allerdings gehört nicht mehr in den Rahmen dieses Beitrages; es soll an anderer Stelle erörtert werden. 23 
1 R. Schnell, "Literarische Beziehungen zwischen Hartmanns Erec und Wolframs Parzival", PBB 95 (Tübingen 1973), S. 301 ff. Zum selben Thema u.a. auch D.H. Green, "Homicide and Parzival", in D.H. Green and L.P. Johnson, Approaches to Wolfram von Eschenbach - Five Essays, Bern, Frankfurt a.M., Las Vegas 1978, S. $11 \mathrm{ff.}$

2 Zwischen Anfang 1979 und Anfang 1980 ist z.B. folgendes erschienen (die Liste erhebt keinerlei Anspruch auf Vollständigkeit!): H. Bayer, "bi den liuten ist so guot. Die meine des Erec Hartmanns von Aue". Euphorion 73 (1979), S. 272 ff.; S.L. Clark, "Changing one's mind: Arenas of conflict in Hartmann's Iwein", Euphorion 73 (1979), S. 286 ff.; O. Ehrismann, "Enite. Handlungsbegründung in Hartmanns von Aue Erec", ZfdPh 98 (1979), S. 321 ff.; U. Ernst, "Der Antagonismus von vita carnalis und vita spiritualis im Gregorius Hartmanns von Aue" (2. Teil.), Euphorion 73 (1979), S. 1 ff.; W. Haug, "Erec, Enite und Evelyne B.", in Medium Aevum 'deutsch', Festschrift für Kurt Ruh zum 65. Geburtstag, Tübingen 1979, S. 139 ff.; G. Kaiser, "Iwein oder Laudine?", ZfdPh 99 (1980), S. 20 ff.; K. Kunze, "Arme Heinrich - Reminiszenz in Ovid-Glossen-Handschrift", ZfdA 63 (1979), S. 31 ff.; W.C. McDonald, "The Maiden in Hartmann's Armen Heinrich : Enite redux?", DtVjs 53 (1979), S. 35 ff.; A. Schirokauer - P. Tax, Hartmann von Aue. Das Büchlein, Berlin 1979.; H. Zutt, König Artus, Iwein, Der Löwe. Die Bedeutung des gesprochenen Wortes in Hartmanns Iwein, Tübingen 1979; H. Mayer, "ein vil vriuntlichez spil: Erecs und Enites gemeinsame Schuld", in Analecta Helvetica et Germanica, Festschrift zu Ehren von Hermann Boeschenstein, Bonn 1979, S. 8 ff.; H. Ragotzky und B. Weinmayer, "Höfischer Roman und soziale Identitätsbildung. Zur soziologischen Deutung des Doppelwegs im Iwein Hartmanns von Aue", sowie A. Hruby, "Hartmann als artifex, philosophus und praeceptor der Gesellschaft". Die beiden letzten Aufsätze in Deutsche Literatur im Mittelalter: Kontakte und Perspektiven. Hugo Kuhn zum Gedenken, Stuttgart 1979, S. 211 ff. und S. $254 \mathrm{ff}$.

3 Vgl. u.a. W. Kellermann, "Die Bearbeitung des Erec-und-Enide - Romans Chrestiens von Troyes durch Hartmann von Aue" (1970), in Hartmann von Aue, hg. von H. Kuhn und C. Cormeau (Wege der Forschung 359), Darmstadt 1973, S. 511 ff.; D.H. Green, "Hartmanns Ironic Praise of Erec", MLR 70 (1975), S. 795 ff.; R. Fisher, "Erecs Schuld und Enites Unschuld bei Hartmann", Euphorion 69 (1975), S. 160 ff.; H. Wenzel, Frauendienst und Gottesdienst. Studien zur Minne-Ideologie, Berlin 1974, S. 173.

4 Vgl. besonders M.H. Jones, "Parzival's Fighting and his Election to the 
Grail", in Wolfram-Studien III, Berlin 1975, S. 52 ff. "Throughout this progress to the peaks of worldly success Parzival has little cause to reflect on the manner in which he achieves his goals, so natural is it to him to win what he wants by deeds of arms." (S. 58)

5 M.K. Jones (vgl. Anm. 4), S. 65: "Instead of being the support of pride and the means of securing incontrovertible rights to whatever he wants, (his fighting) is coupled with humility and is a means of showing merit which will make him worthy to be granted the prizes to which he aspires".

6 Aggressivität spielt natürlich auch im Erec eine sehr wichtige Rolle, vgl. u.a. D.H. Green, "Homicide and Parzival" (vgl. Anm. 1), Erec wendet sie aber nicht in unangebrachter Weise an, wie es Parzival z.B. in der Jeschute-Episode tut.

7 Vgl. H. Adolf, "Structure and Character Delineation in the Parzival", in Wolfram-Studien V, Berlin 1979, S. $166 \mathrm{ff}$. "Parzival . . fails to notice the mixture of anger and mirth in the behavior of the jester; he is still a very young dog indeed". (Adolf, S. 169). Die wichtige Tatsache, daß Parzival hier 'entblößt', ohne Rüstung und Waffen dasteht, kommentiert D.H. Green, "The Concept aventiure in Parzival", in Approaches to Wolfram von Eschenbach (vgl. Anm. 1), S. 83 ff., hier S. 150 f.

$8 \mathrm{Zu}$ meiner Interpretation der Ehekrise im Erec siehe K. Smits, "Enite als christliche Ehefrau", in Festschrift für John Asher zum 60. Geburtstag, Berlin 1981 (im Druck). Es wird hier die Rolle der Ehefrau im Lichte des frühscholastischen Frauenbildes und der frühscholastischen Ehetheorie untersucht. Vgl. auch D.H. Green, "The Concept aventiure" (vgl. Anm. 7). In Bezug auf Parzival und Sir Gawain and the Green Knight (und besonders im Hinblick auf Parzivals Neigung, jedes Problem durch ritterlichen Kampf lösen zu wollen) schreibt Green (S. 153): "The German and English authors both conduct their heroes (and their listeners) to the unexpected recognition that their narrative, whilst illustrating the very real merits of chivalric adventure, has also been at work in cutting its pretensions down to size by critically defining its limitations". Dasselbe dürfte auch schon für Hartmann zutreffen.

9 R. Schnell (vgl. Anm. 1), S. 323 ff.

10 Zur Guivreiz-Gestalt siehe u.a. D.H. Green, "Homicide and Parzival" (vgl. Anm. 1), S. 19 ff. und die dort angeführte Literatur.

11 Siehe dazu K. Smits, "Enite als christliche Ehefrau" (vgl. Anm. 8).

12 Vgl. Anm. 11. Eine wichtige Aufgabe der Frau ist es, der Ehe als 'Auge und Ohr' zu dienen.

13 Vgl. H. Kuhn, Erec (1948), in Wege der Forschung 359 (vgl. Anm. 3), 
S. 17 ff. (hier S. 45, Anm. 73) und K. Smits, "Enite als christliche Ehefrau" (vgl. Anm, 8).

14 boesen (4335) bezieht sich natürlich auf den gesellschaftlichen Rang des Mannes, dadurch aber auch auf dessen Wert als Person.

15 R. Schnell (vgl. Anm. 1), S. 304 ff. Zur Parzival-Ehe siehe besonders M. Schumacher, Die Auffassung der Ehe in den Dichtungen Wolframs von Eschenbach, Heidelberg 1967.

16 Vgl. Anm. 11.

17 R. Schnell (vgl. Anm. 1), besonders S. 322.

18 R. Schnell (vgl. Anm. 1), S. 312 ff.

19 Siehe dazu K. Smits, "Die Schönheit der Frau in Hartmanns Erec" (erscheint 1981 in $\mathrm{ZfdPh}$ ).

20 Daß Turnier und Kampf im Erec unterschiedliche Funktionen haben, wird unterstrichen durch die Beobachtung D.H. Greens ("Homicide and Parzival", vgl. Anm. 1), "that Wolfram, unlike Hartmann, was quite prepared to indicate that even the tournament, a well-controlled and stylised form of combat, could in reality degenerate into a rough-and-tumble in which tempers were lost and grievous, even mortal wounds could be inflicted." (S. 34). "This is quite different from Hartmann's stylised idealization of the tournament in Erec to celebrate the hero's wedding." (S. 35).

21 Vgl. Anm. 19.

22 R. Schnell (vgl. Anm. 1), S. 314 ff. Während im Erec die Bewältigung der christlichen Ehe ein Hauptmotiv ist (vgl. Anm. 6), liegen die Aufgaben Parzivals auf einer anderen Ebene.

23 Vgl. Anm. 19. 